Research paper

\title{
Correlates of a general psychopathology factor in a clinical sample of childhood sexual abuse survivors
}

\author{
Philip Hyland ${ }^{\mathrm{a}, \mathrm{b}, *}$, Jamie Murphy ${ }^{\mathrm{c}}$, Mark Shevlin ${ }^{\mathrm{c}}$, Steven Carey $^{\mathrm{c}}$, Frédérique Vallières ${ }^{\mathrm{b}}$, \\ David Murphy ${ }^{\mathrm{b}}$, Ask Elklit ${ }^{\mathrm{d}}$

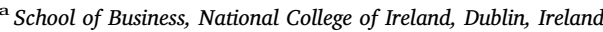 \\ bentre for Global Health, School of Psychology, Trinity College Dublin, Dublin, Ireland \\ ${ }^{c}$ Psychology Research Institute, School of Psychology, Ulster University, Derry, Northern Ireland \\ d National Centre for Psychotraumatology, School of Psychology, University of Southern Denmark, Odense, Denmark
}

\begin{abstract}
A B S T R A C T
Background: Childhood sexual abuse (CSA) increases risk for most psychiatric disorders. There is evidence that the structure of psychopathology can be explained by a number of latent dimensions of psychopathology including a 'General Psychopathology' (P) factor. The objective of the current study was to provide the first assessment as to whether $\mathrm{P}$ is identifiable, and what its correlates might be in a clinical sample.

Methods: An adult, clinical sample of Danish CSA survivors $(N=420)$ was assessed using the Millon Clinical Multiaxial Inventory-III. Confirmatory factory analysis (CFA) was used to assess the latent structure of nine psychiatric disorders, and structural equation modelling (SEM) was used to determine correlates of the bestfitting dimensional model.

Results: CFA results favoured a bifactor model including three specific dimensions of psychopathology, "Internalizing", "Externalizing", and "Thought Disorder", and a bi-factor "P". A SEM model that included ten predictors was a good fit to the data and explained $55 \%$ of variance in ' $\mathrm{P}$ '. The 'P' factor was significantly associated with emotional coping, negative self-worth, traumatic life events, and anxious attachments.

Limitations: Psychiatric disorders were assessed using self-report measures, and the sample was predominately female.

Conclusions: Results provide initial evidence of $\mathrm{P}$ in a clinical sample and several unique correlates of this factor were identified.
\end{abstract}

\section{Introduction}

Childhood sexual abuse (CSA) has been consistently demonstrated to increase risk of most psychiatric disorders (Molnar et al., 2001) and diagnostic comorbidity (Owens and Chard, 2003). In a household survey of the English population (Jonas et al., 2011), CSA increased risk of common internalizing (e.g., depression, anxiety, posttraumatic stress disorder (PTSD)), and externalizing (drug dependence, and alcohol dependence) disorders. Additionally, multiple studies based on nationally representative and clinical samples have demonstrated that CSA increases risk of psychotic disorders such as bipolar and schizophrenia (e.g. (Bebbington, 2009; Bendall et al., 2008; Murphy et al., 2014)). Taken together, the existing literature suggests that exposure to a specific early traumatic life event (i.e., CSA) is a risk-factor for psychopathology in general.

Such findings are interesting to consider in light of a growing literature criticising the validity of the categorical model of psychopathology as presented within diagnostic nosologies such as the Diagnostic and Statistical Manual of Mental Disorders (DSM) (American Psychological Association, 2013) and the International Classification of Diseases (ICD) (World Health Organization, 1992). The categorical model suggests that psychopathological symptoms can be reliably and validly partitioned into discrete independent psychiatric disorders, which themselves can be meaningfully differentiated. However, substantial factor analytic research evidence has undermined this assumption and has instead indicated that psychological distress can be more accurately and parsimoniously understood in terms of a small number of latent continuous factors which explain the covariation between diagnoses, and the symptoms that comprise these diagnoses (Lahey et al., 2017).

More recently, a consortium of clinical researchers synthesised the existing empirical literature regarding the latent structure of

\footnotetext{
* Correspondence to: School of Business, National College of Ireland, IFSC, Mayor Street, Dublin 1, Ireland.

E-mail address: Philip.hyland@ncirl.ie (P. Hyland).
} 
psychopathology and presented a new dimensional model of psychopathology called the 'Hierarchical Taxonomy of Psychopathology' (HiTOP: (Kotov et al., 2017). Factor analytic research with clinical and community samples of adults, adolescents, and children provides strong evidence for three fundamental dimensions of psychopathology: 'Internalizing' (reflecting difficulties with emotion regulation and negative affectivity), 'Externalizing' (reflecting difficulties with behavioural inhibition), and 'Thought Disorder' (reflecting difficulties with perceptions of reality) (Fleming et al., 2014; Forbush and Watson, 2013; Kotov et al., 2011a; Kotov et al., 2011a, b; Krueger and Markon, 2011; Wright et al., 2013). There is also burgeoning evidence of a 'Somatoform' dimension which reflects problematic responses to bodily related symptoms (Kotov et al., 2011b; McNulty and Overstreet, 2014; Sellbom, 2017). In addition to these circumscribed dimensions of psychopathology, there is also strong evidence indicating the presence of a 'General Psychopathology' (P) factor which accounts for covariation across all forms of psychopathology (Carragher et al., 2016; Caspi et al., 2014; Lahey et al., 2012; Patalay et al., 2015; Tackett et al., 2013). This $\mathrm{P}$ factor has been identified in multiple adult community-based samples (Caspi et al., 2014; Lahey et al., 2012; Martel et al., 2017), as well as child and adolescent community samples (Carragher et al., 2016; Laceulle et al., 2015; Lahey et al., 2015; Martel et al., 2017; Patalay et al., 2015; Tackett et al., 2013; Waldman et al., 2016). Notably however, no study has yet investigated if $\mathrm{P}$ is identifiable within a clinical sample.

The exact nature $\mathrm{P}$ is not well understood as few studies have assessed its external correlates. When initial evidence of $\mathrm{P}$ was identified, Lahey et al. (2012) acknowledged that it might simply have been a statistical artefact arising from the common method variance shared across disorders, or as a result of the same respondents and measures being used to obtain the data. However, since this initial finding several studies have established the criterion and predictive validity of $\mathrm{P}$, as well as its temporal stability, suggesting that $\mathrm{P}$ is likely a valid psychological construct. Amongst a birth cohort of the New Zealand adult population who were for tracked across a 20 -year period, Caspi et al. (2014) found that P was: (i) temporally stable in adulthood, (ii) positively correlated with neuroticism, and negatively correlated with agreeableness, conscientiousness, and IQ scores, and (iii) predicted future risk of suicidal attempts, psychiatric hospitalization, duration of time spent on social welfare, and criminal conviction for violent conduct. In an English adolescent sample, Patalay et al. (2015) found that P predicted future emotional and behavioural difficulties, and poorer academic performance in school. Additionally, the predictive effects of $\mathrm{P}$ were greater than those observed for all other dimensions of psychopathology. One limitation of Patalay et al.'s findings was that they were based on the use of factor scores, which can lead to inaccurate estimates of association relative to the true latent variable score (Muthén, 2007). Overcoming these limitations by the use of structural equation modelling (SEM), Carragher et al. (2016) found strong associations between $\mathrm{P}$ and negative thinking and impulsivity. Carragher et al. also reported that females scored significantly higher on the Internalizing dimension, significantly lower on the Externalizing dimension, and that there were no sex differences on the $\mathrm{P}$ and Thought Disorder dimensions.

The objective of the current study is to undertake the first assessment of whether $P$ is identifiable within a clinical sample, and what its external correlates may be. The current clinical sample are characterised by exposure to CSA and sought treatment for psychological distress associated with this early life trauma. Given that CSA is a known risk-factor for diagnoses across the Internalizing, Externalizing, and Thought Disorder dimensions, it is valuable to analyse sample data characterised by such an established risk-factor for all forms of psychopathology. This affords a unique opportunity to test whether dimensional models of psychopathology, derived from general population data, can accurately capture the covariation between psychiatric disorders within a more constrained, risk-orientated context.

\subsection{Aims of the study}

The first aim of the current study was to assess the latent structure of nine common psychiatric disorders (anxiety, dysthymia, PTSD, depression, alcohol dependence, drug dependence, bipolar disorder, delusional disorder, and thought disorder) using confirmatory factor analysis (CFA). Based on the predictions of the HiTOP model, it was hypothesised that a bifactor model which included an Internalizing factor (measured by anxiety, dysthymia, PTSD, and depression), an Externalizing factor (measured by alcohol dependence, and drug dependence), a Thought Disorder factor (measured by bipolar disorder, delusional disorder, and thought disorder), and a P factor (measured by all nine disorders) would provide optimal fit to the data. The second aim of the study was to use SEM to evaluate the unique correlations between several demographic (sex, age), trauma-related (number of lifetime traumatic exposures), and psychological (coping styles, attachment styles, and self-worth) variables and $\mathrm{P}$. Assuming that $\mathrm{P}$ is a valid psychological construct, it was hypothesised that $\mathrm{P}$ would be significantly correlated with several criterion variables.

\section{Method}

\subsection{Participants and procedure}

Participants were attendees, predominately female (85\%), of four treatment centres in Denmark that provide psychological treatment for victims of CSA $(N=420)$. Clients were asked to complete a series of self-report questionnaires during their second visit to the treatment centre, and therapy is structured around responses to these assessments. The present study is based on information from these questionnaires. Most of the participants (91\%) had experienced CSA before the age of 15 , committed by a person at least five years older. The mean age of the sample was 36.40 years $(S D=10.80$; range $18-70$ years). All participants were Caucasian; $51 \%$ were married or cohabiting; $59 \%$ had children, and the average length of education was 13.31 years (SD $=3.32$; range 7-24 years). Ethical approval for this study was provided by the ethical review board to which the final author is attached.

\subsection{Measures}

\subsubsection{Psychiatric disorders}

Psychiatric disorders were measured using the Millon Clinical Multiaxial Inventory-III (Millon et al., 2009) The MCMI-III is a self-report tool intended to provide information on psychopathology, including specific disorders outlined in the DSM-IV (American Psychological Association, 1994). The scale measures ten psychiatric disorders (anxiety, somatoform disorder, ${ }^{1}$ dysthymia, PTSD, major depression, alcohol dependence, drug dependence, bipolar disorder, delusional disorder, and thought disorder). It is intended for adults (18 and over) with at least an eighth grade reading level, currently seeking mental health services. The MCMI was developed and standardised specifically for use with clinical populations (i.e., patients in psychiatric hospitals or people with existing mental health problems). The MCMI-III includes 175 'true' (1) or 'false' (0) questions that can generate 'raw scores' or 'base rate' scores for each disorder. Raw scores are continuously measured variables where higher scores indicating higher levels of each psychiatric disorder. Raw scores were used for the purpose of the current modelling, but can also be converted into base rate scores for the purposes of calculating diagnosis. The MCMI-III was translated into Danish and Elklit (2004) demonstrated the discriminative validity of the Danish MCMI-III in the analyses of a number of patient groups.

\footnotetext{
${ }^{1}$ For the purposes of the present study somatoform disorder was disregarded as the HiTOP model presents Somatoform as a separate dimension of psychopathology, and this dimension could not be modelled with just one measurement.
} 
Scale intercorrelations were similar across the Danish and the US samples, and the range of Cronbach's alpha $(\alpha)$ values of the MCMI-III scales (.64-.93) of the Danish sample was comparable to the range of values (.66-.95) in the MCMI-III manual (Millon et al., 2009).

\subsubsection{Coping styles}

The Coping Style Questionnaire (CSQ) originally consisted of 60 items (4-point Likert scale; $1=$ 'never', $4=$ 'always') and measured four types of (adaptive and maladaptive) coping: (i) rational coping (adaptive: attempts to solve the problem), (ii) emotion-focused coping (maladaptive: focus on the negative emotions associated with the problem and one's helplessness to solve the problem), (iii) avoidant coping (maladaptive: pretending that the problem doesn't exist and ignoring it), and (iv) detached coping (adaptive: viewing the problem in a realistic light and not identifying with the problem) (Roger et al., 1993). Higher scores on each subscales reflect higher levels of each coping style. The current sample was assessed using a shorter, 37-item version of the CSQ developed and validated within a Danish sample by Elklit, (1996). The internal reliabilities of the rational ( $\alpha=.74)$, and emotionfocused ( $\alpha=.84$ ) subscales were satisfactory, while the reliabilities of the avoidant $(\alpha=.62)$ and detached $(\alpha=.63)$ subscales were somewhat lower.

\subsubsection{Attachment styles}

The Revised Adult Attachment Scale (RAAS) (Collins, 1996) is an 18item, self-report measure that generates scores of two attachment styles: (i) Anxious attachment (6 items: $\alpha=.49$ ) and (ii) Avoidant attachment (12 items: $\alpha=.74$ ). Respondents indicate how they normally function and feel in a relationship with a partner, someone close, and people in general using a five-point Likert scale $(1=$ 'not at all characteristic' to 5 'very characteristic'). The psychometric properties of the RAAS amongst a Danish sample have previously been evidenced (O'Connor and Elklit, 2008).

\subsubsection{Negative self-worth}

Negative self-worth was measured using this subscale of the World Assumptions Scale (WAS) (Janoff-Bulman, 1989). The 32-items of the WAS were originally designed to capture three subscales (Benevolence of the World, Meaningfulness of the World, Worthiness of the Self) however several psychometric assessments have suggested the presence of additional factors. In an assessment of a Danish sample, Elklit et al. (2007) found that an eight-factor model best explained the structure of the WAS. One of these factors reflected Negative Self-Worth and this scale was measured via four items (six-point Likert scale, $1=$ 'strongly disagree' to $6=$ 'strongly agree'). The reliability of this subscale was satisfactory $(\alpha=.77)$.

\subsubsection{Traumatic exposure}

Respondents were asked to indicate "if in their lifetime they had ever been exposed to" any of 12 types of trauma (e.g., physical abuse, neglect, war). Responses were based on a 'Yes' (1) or 'No' (0) format generating a total possible score ranging from 0 to 12 .

\subsection{Analysis}

Six different dimensional models of psychopathology, derived from the existing literature, were assessed using CFA and confirmatory bifactor analysis (see Fig. 1). Model 1 is a correlated three-factor model (Internalizing, Externalizing, and Thought Disorder). Model 2 is a bifactor model that includes a $\mathrm{P}$ factor in addition to the three factors in Model 1. Model 3 is similar to Model 1 but separates the Internalizing dimension into two sub-factors of Fears and Distress (Lahey et al., 2012). Model 4 is a bifactor model that includes $P$ in addition to the four factors in Model 2. Model 5 is a replication of the model favoured by Caspi et al. (2014) in which the Thought Disorder dimension is removed. Finally, Model 6 is a one-factor model in which the covariations between all psychiatric disorders are explained by the P factor alone.

Upon selection of the best fitting measurement model of psychopathology, a SEM model was developed and tested. This model included ten predictor variables: sex (Males $=0$, Females $=1$ ), age, total lifetime traumatic exposures, rational coping, emotion-focused coping, avoidant coping, detached coping, anxious attachment, avoidant attachment, and negative self-worth. All predictor variables were treated as observed variables in the model. The latent psychopathology factors of the best fitting measurement model were regressed simultaneously onto each predictor variable to determine the unique associations between each predictor variable and each dimension of psychopathology.

The CFA and SEM models were tested using Mplus version 7.4 (Muthen and Muthen, 2013) using robust maximum likelihood (MLR) estimation (Yuan and Bentler, 2000). MLR is desirable due to the fact that it is robust to non-normally distributed data and can produce corrected standard errors under conditions of non-normality (Enders and Bandalos, 2001). MLR also allows parameters to be estimated using all available information and is thought to be superior to alternative methods of handling missing data, such as listwise deletion (Schafer and Graham, 2002). Good model fit was indicated by a chi-square-todegree of freedom ratio of less than 3:1; Comparative Fit Index (CFI) and Tucker Lewis Index (TLI) values $>.95$ indicate excellent fit and values $>.90$ indicate acceptable fit; and Root-Mean-Square Error of Approximation with 90\% confidence intervals (RMSEA 90\% CI) and Standardized Root-Mean-Square Residual (SRMR) values $<.05$ indicate excellent fit and values $<.08$ indicate acceptable fit. The Bayesian Information Criterion (BIC) was used to evaluate alternative nested and non-nested models and the model with the smallest value is considered to be the best fitting model. A 10-point difference between two BIC values has been suggested to represent strong evidence (odds ratio 150:1) that the model with the lower value is superior (Raftery, 1995). The CFI, RMSEA, and BIC all have explicit penalties for model complexity.

\section{Results}

\subsection{Prevalence rates and correlations between the psychiatric disorder}

Table 1 lists the prevalence rates for each psychiatric disorder based on the MCMI-III. The most common diagnosis was anxiety (76\%) and the least common diagnosis was drug dependence (4\%). The correlations between the nine disorders are reported in Table 2. All correlations were statistically significant $(p<.001)$ and the associations within dimensions (Internalizing, Externalizing, and Thought Disorder) were generally stronger than the associations across dimensions.

\subsection{Latent structure of psychopathology}

The model fit results for the six dimensional models of psychopathology are reported in Table 3. All models estimated normally with the exception of Model 4 which was rejected due to the occurrence of a number of correlations $>1.0$ between the Distress factor and several other factors in the model. Models 1, 3, 5, and 6 were rejected as poor approximations of the data. Model 2 possessed excellent fit across all indices indicating extremely close fit to the sample data. This model, which included Internalizing, Externalizing, Thought Disorders, and P, was judged to be the best representation of the latent structure of the nine psychiatric disorders.

The standardised factor loadings, and factor correlations, are reported in Table 4. The nine disorder loaded onto $\mathrm{P}$ positively, statistically significantly $(\mathrm{p}<.001$ ), and robustly (mean factor loading $=.61$ ). Each of the Internalizing disorders loaded more strongly on $\mathrm{P}$ than they did on the Internalizing factor. Alcohol dependence and drug dependence loaded more strongly on the Externalizing factor than they did on P. Delusional disorder and thought disorder loaded more strongly on P than they did on the Thought Disorder factor, whereas 
Model 1.

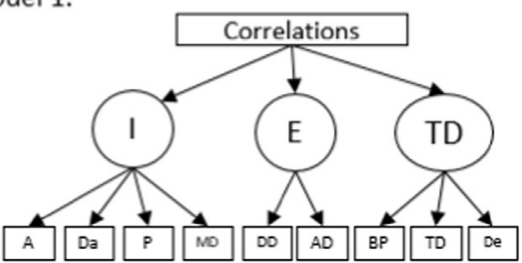

Model 3.

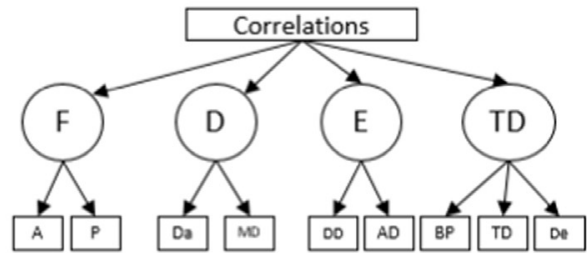

Model 5.

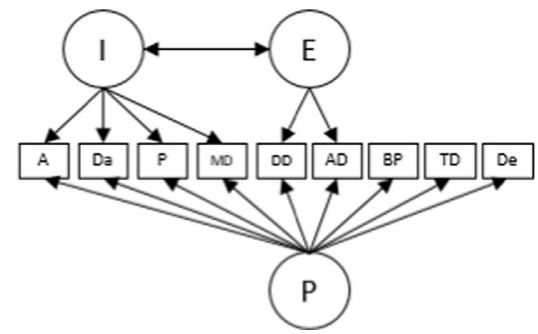

Model 2.

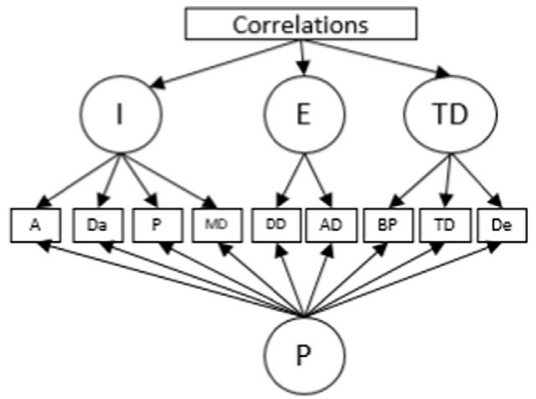

Model 4.

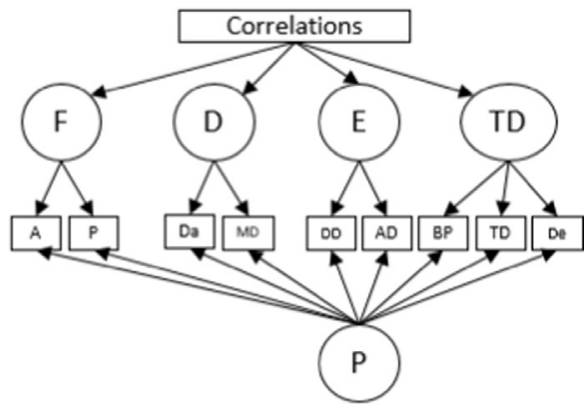

Model 6.

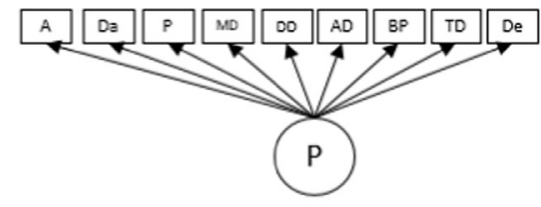

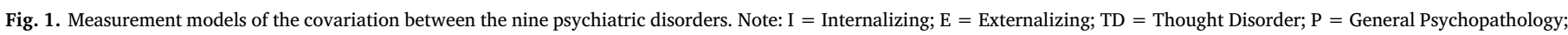

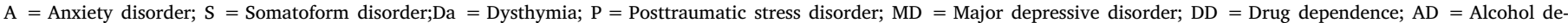
pendence; $\mathrm{BP}=$ Bipolar disorder; $\mathrm{TD}=$ Thought disorder; $\mathrm{De}=$ Delusional Disorder.

Table 1

Prevalence rates for each psychiatric disorder based on the MCMI-III $(\mathrm{N}=420)$.

\begin{tabular}{lll}
\hline & Frequency & $\%$ \\
\hline \multicolumn{1}{l}{ Internalizing Disorders } & & \\
Anxiety & 320 & 76 \\
Dysthymia & 164 & 39 \\
PTSD & 87 & 21 \\
Depression & 156 & 37 \\
$\quad$ Externalizing Disorders & & \\
Alcohol dependence & 22 & 5 \\
Drug Dependence & 15 & 4 \\
$\quad$ Thought Disorders & & \\
Bipolar Disorder & 48 & 11 \\
Thought disorder & 72 & 17 \\
Delusional disorder & 34 & 8 \\
\hline
\end{tabular}

bipolar disorder loaded more strongly on the Thought Disorder factor than it did on P. The Internalizing factor was uncorrelated with the Externalizing factor $(r=.04)$, and was weakly correlated with the Thought Disorder factor $(r=.30)$. The relationship between the Externalizing and Thought Disorder factors was positive and strong $(\mathrm{r}=.66)$.

\subsection{SEM results}

The SEM model (see Table 5 for full details) which included four
Table 2

Correlations between scores of each psychiatric disorder as measured by the MCMI-III.

\begin{tabular}{llllllllll}
\hline & 1 & 2 & 3 & 4 & 5 & 6 & 7 & 8 & 9 \\
\hline 1. Anxiety & 1 & & & & & & & & \\
2. Dysthymia & .55 & 1 & & & & & & & \\
3. PTSD & .83 & .63 & 1 & & & & & & \\
4. Depression & .62 & .87 & .66 & 1 & & & & & \\
5. Alcohol Dependence & .25 & .31 & .27 & .29 & 1 & & & & \\
6. Drug Dependence & .19 & .19 & .22 & .22 & .57 & 1 & & & \\
7. Bipolar Disorder & .34 & .20 & .27 & .20 & .44 & .42 & 1 & & \\
8. Thought Disorder & .69 & .78 & .70 & .75 & .43 & .33 & .47 & 1 & \\
9. Delusional Disorder & .44 & .37 & .44 & .37 & .32 & .32 & .41 & .49 & 1 \\
\hline
\end{tabular}

Note: All correlations are statistically significant $(\mathrm{p}<.001)$.

latent factors of psychopathology and ten observed predictor variables provided a good fit to the data $(\chi 2=102.944$, $\mathrm{df}=65, \mathrm{p}=.002$; CFI $=.979 ;$ TLI $=.960 ;$ RMSEA $=.047[90 \% \mathrm{CI}=.029-.063]$; SRMR $=.026)$. The model explained $55 \%$ of variance in $\mathrm{P}, 16 \%$ of variance in Internalizing, $27 \%$ of variance in Externalizing, and $22 \%$ of variance in Thought Disorder.

The $\mathrm{P}$ factor was most strongly correlated with emotion-focused coping $(\beta=.42, p<.001)$, followed by negative self-worth $(\beta=.26$, $\mathrm{p}<.001)$, traumatic life events $(\beta=.12, \mathrm{p}<.05)$, and anxious attachments $(\beta=-.11, \mathrm{p}<.05)$. The Internalizing factor was correlated with female sex $(\beta=.22, p<.001)$, traumatic life events $(\beta=.22, \mathrm{p}<.001)$, and younger age $(\beta=-.13, \mathrm{p}<.05)$. The 
Table 3

Model fit results for the alternative dimensional models of the structure of psychopathology as measured by the MCMI-III.

\begin{tabular}{llllllll}
\hline Models & $\chi^{2}$ & $d f$ & CFI & TLI & RMSEA (90\% CI) & SRMR & BIC \\
\hline $\begin{array}{l}\text { Model 1 } \\
\text { (I, E, TD) }\end{array}$ & $462.658^{*}$ & 24 & .807 & .711 & $.209(.192-.225)$ & .082 & 20126 \\
$\begin{array}{l}\text { Model 2 } \\
\text { (I, E, TD, P) }\end{array}$ & 17.728 & 15 & .999 & .997 & $.021(.000-.053)$ & .013 & 19725 \\
$\begin{array}{l}\text { Model 3 } \\
\quad \text { F, D, E, TD) }\end{array}$ & $177.482^{*}$ & 21 & .931 & .882 & $.133(.115-.152)$ & .068 & 19857 \\
$\begin{array}{l}\text { Model 4 } \\
\text { (F, D, E, TD, P) }\end{array}$ & - & - & - & - & - & & \\
$\begin{array}{l}\text { Model 5 (I, E, P) } \\
\text { Model 6 (P) }\end{array}$ & $388.354^{*}$ & 21 & .839 & .723 & $.204(.187-.222)$ & .079 & 20069 \\
& $650.998 *$ & 27 & .726 & .635 & $.235(.219-.250)$ & .107 & 20328 \\
\hline
\end{tabular}

Note. $\quad N=420 ; \mathrm{I}=$ Internalizing; $\mathrm{E}=$ Externalizing; $\mathrm{TD}=$ Thought Disorder; $\mathrm{P}=$ General Psychopathology; $\mathrm{D}=$ Distress; $\mathrm{F}=$ Fears; $\chi^{2}=$ chi square goodness of fit statistic; $d f=$ degrees of freedom; CFI $=$ Comparative Fit Index; TLI $=$ Tucker Lewis Index; RMSEA $(90 \% \mathrm{CI})=$ Root-Mean-Square Error of Approximation with $90 \%$ confidence intervals; SRMR $=$ Standardized Square Root Mean Residual; BIC $=$ Bayesian Information Criterion

* Indicates $\chi^{2}$ are statistically significant $(p<.001)$; Model 5 did not estimate normally due to correlations greater than 1 .

Table 4

Standardized factor loadings (standard errors) and correlation (standard errors) for General Psychopathology (P), Internalizing, Externalizing, and Thought Disorder.

\begin{tabular}{|c|c|c|c|c|}
\hline Item & $\mathrm{P}$ & Internalizing & Externalizing & $\begin{array}{l}\text { Thought } \\
\text { Disorder }\end{array}$ \\
\hline \multicolumn{5}{|l|}{ Factor Loadings } \\
\hline Anxiety & $.75(.03)$ & $.62(.06)$ & & \\
\hline Dysthymia & $.94(.01)$ & $-.25(.05)$ & & \\
\hline PTSD & $.78(.03)$ & $.40(.04)$ & & \\
\hline Depression & $.90(.01)$ & $-.10(.04)^{*}$ & & \\
\hline Alcohol Dependence & $.33(.04)$ & & $.71(.05)$ & \\
\hline Drug Dependence & $.22(.04)$ & & $.71(.04)$ & \\
\hline Bipolar: Mania & $.27(.05)$ & & & $.76(.04)$ \\
\hline Thought Disorder & $.85(.01)$ & & & $.40(.05)$ \\
\hline Delusional Disorder & $.44(.05)$ & & & $.32(.03)$ \\
\hline \multicolumn{5}{|l|}{ Factor } \\
\hline Internalizing & & & $.04(.07)^{k . * 8}$ & $.30(.07)$ \\
\hline Externalizing & & & & $.66(.05)$ \\
\hline
\end{tabular}

Note. All factor loadings are statistically significant $(\mathrm{p}<.001)$ except.

$*(\mathrm{p}=.03)$, and.

$* *(\mathrm{p}=.60)$.
Externalizing factor was correlated with female gender $(\beta=-.30$, $\mathrm{p}<.001)$, traumatic life events $(\beta=.20, \mathrm{p}<.05)$, and avoidant coping $(\beta=.19, \mathrm{p}<.05)$. The Thought Disorder factor was correlated with avoidant coping $(\beta=.37, \mathrm{p}<.001)$, and anxious attachment style $(\beta=.15, \mathrm{p}<.05)$.

\section{Discussion}

Current findings which provide empirical support for the validity of a general factor of psychopathology within a Danish, clinical sample of CSA survivors align with findings from child, adolescent, and adult community-based samples from Australia (Carragher et al., 2016), Brazil (Martel et al., 2017), England (Patalay et al., 2015), New Zealand (Caspi et al., 2014), the Netherlands (Laceulle et al., 2015), and the United States (Lahey et al., 2012). Prior findings demonstrate the continuity of the latent structure of psychopathology across the lifespan, and the consistency in the latent structure of psychopathology across cultures. However, a central prediction of the HiTOP model (or any dimensional model of psychopathology) is that psychopathology exists on a continuum that is distributed across the population. Consequently, the latent structure of psychopathology should remain consistent across clinical and community samples. The current findings demonstrate for the first time that the latent structure of psychopathology amongst a clinical sample is indeed congruent with what has previously been observed in community samples. This represents important supportive evidence for the dimensional representation of psychopathology.

The results of this study provide additional evidence that the latent structure of common psychiatric disorders can be explained by the dimensions of Internalizing, Externalizing, Thought Disorder, and P. This model provided an excellent representation of the sample data and it was notable that six of the nine disorders were most strongly related to the $\mathrm{P}$ factor. This suggests that risk of diagnosis for the majority of the psychiatric disorders assessed in the current study was primarily dependent on one's levels of P. That each Internalizing disorder was more strongly associated with $\mathrm{P}$ is consistent with findings from several other studies (Caspi et al., 2014; Lahey et al., 2012; Patalay et al., 2015). Contrastingly, alcohol dependence and drug dependence were most strongly related to the Externalizing dimension. The existing literature is contradictory in this regard as some studies have found that the diagnoses/symptoms that comprise the Externalizing dimension are more strongly related to this dimension than to P (Lahey et al., 2012; Patalay et al., 2015), while others have found the opposite relationship (Carragher et al., 2016; Caspi et al., 2014). The inconsistent findings may be attributable to the limited number of diagnoses, varied

Table 5

Standardized regression coefficients $(\beta)$ and standard errors (SE) for each predictor variable on each dimension of psychopathology.

\begin{tabular}{|c|c|c|c|c|}
\hline & $\begin{array}{l}P \\
\beta(S E)\end{array}$ & $\begin{array}{l}\text { Internalizing } \\
\beta \text { (SE) }\end{array}$ & $\begin{array}{l}\text { Externalizing } \\
\beta \text { (SE) }\end{array}$ & $\begin{array}{l}\text { Thought Disorder } \\
\beta \text { (SE) }\end{array}$ \\
\hline Gender (Female) & $-.01(.04)$ & $.22(.06)^{* * * * x}$ & $-.30(.08)^{* * * *}$ & $-.07(.07)$ \\
\hline Age & $-.06(.05)$ & $-.13(.07)^{*}$ & $-.09(.07)$ & $-.07(.07)$ \\
\hline Number of lifetime traumas & $.12(.05)^{* * *}$ & $.22(.06)^{k+k \times k}$ & $.20(.08)^{*}$ & $.05(.08)$ \\
\hline Rational Coping Style & $-.07(.05)$ & $.12(.07)$ & $.04(.09)$ & $.16(.09)$ \\
\hline Emotion-focused Coping Style & $.42(.06)^{n+k}$ & $-.10(.09)$ & $-.12(.11)$ & $-.06(.12)$ \\
\hline Avoidance Coping Style & $.08(.05)$ & $.12(.08)$ & $.19(.08)^{*}$ & $.37(.07)^{k .3 .4}$ \\
\hline Detached Coping Style & $.00(.06)$ & $-.10(.07)$ & $.17(.09)$ & $.06(.09)$ \\
\hline Avoidance Attachment Style & $.08(.05)$ & $-.05(.05)$ & $.11(.08)$ & $-.11(.09)$ \\
\hline Anxious Attachment Style & $-.11(.05)^{*}$ & $-.03(.07)$ & $.08(.07)$ & $.15(.07)^{*}$ \\
\hline Negative Self-Worth & $.26(.06)^{k \ldots \ldots k t}$ & $-.12(.09)$ & $.16(.10)$ & $.04(.10)$ \\
\hline $\mathrm{R}^{2}$ & $.55^{n+\infty x+x}$ & $.16^{* * * x}$ & $.27^{\text {*ै* }}$ & $.22^{2 * k \times 2}$ \\
\hline
\end{tabular}

Note: $\mathrm{P}=$ General Psychopathology; $\mathrm{R}^{2}=$ Percentage of variance explained.

$* \mathrm{p}<.05$.

$* * \mathrm{p}<.01$.

$* * * \mathrm{p}<.001$. 
symptom indicators, or the stage of development (adolescent or adult) of the sample participants. Two of the three Thought Disorder diagnoses were more strongly associated with $\mathrm{P}$ in the current sample. There is scant evidence for the relative strength of associations between psychotic disorders/symptoms and the Thought Disorder and P factors, respectively. Carragher et al. (2016) found that the majority of psychotic symptoms loaded slightly more strongly on the Thought Disorder factor as compared to the $\mathrm{P}$ factor however the factor loadings were robust for each dimension. The existing literature indicates that $\mathrm{P}$ is a significant vulnerability factor for all forms of psychopathology however $\mathrm{P}$ appears to elevate risk of diagnosis most strongly along the Internalizing dimension, followed by the Thought Disorder dimension, and finally the lowest (or most variable) risk is associated with the Externalizing dimension.

Repeated identification of a measurement structure that includes a general factor of psychopathology is insufficient evidence for acceptance of $\mathrm{P}$ as a valid construct. To further assess the validity of the $\mathrm{P}$ factor, the dimensional structure of psychopathology was integrated within a SEM model that simultaneously assessed the associations between ten covariates and the $\mathrm{P}$ factor, while accounting for the presence of the Internalizing, Externalizing, and Thought Disorder dimensions. If $P$ were merely a method effect with no substantive meaning, it would be expected that the covariates would correlate meaningfully with the specific dimensions of psychopathology but not with P. However, the SEM findings indicated statistically significant associations between $\mathrm{P}$ and several psychological variables. The strongest correlate of $\mathrm{P}$ was emotion-focused coping strategies. Emotion-focused coping is considered a maladaptive coping strategy whereby an individual's typical response to a stressor is to respond with high levels of negative affectivity. Emotion-focused coping has previously been associated with high levels of neuroticism (Christiansen et al., 2014) and psychological distress (e.g. (Ireland et al., 2005)). P was also associated with increased negative evaluations of oneself, more frequent traumatic exposures, and lower scores of anxious attachments. The ten predictor variables explained $55 \%$ of variance in levels of $P$, which substantially exceeded the variance explained in the other dimensions of psychopathology. These observations support the criterion validity of $P$, and provide further evidence that $\mathrm{P}$ may well be a meaningful psychological construct.

The SEM results also indicated that certain variables were: (i) correlated with $\mathrm{P}$ but uncorrelated with any specific dimension of psychopathology (e.g., emotion-focused coping and negative self-worth); (ii) uncorrelated with $\mathrm{P}$ but correlated with at least one other specific dimension of psychopathology (e.g., the association between avoidant coping and Thought Disorders); and (c) correlated with multiple dimensions of general and specific psychopathology (e.g., traumatic exposure). Despite the restricted number of covariates used in this study, these results highlight the probability that there are both unique riskfactors for specific dimensions of psychopathology, and shared riskfactors that span multiple dimensions of (specific and general) psychopathology. Identification of the unique and shared risk-factors for psychopathology affords the opportunity to develop unique risk-profiles for different forms of psychological distress. These risk-profiles may in turn be used to develop clinical interventions that influence distress within and across dimensions of psychopathology. Additionally, such risk-profiles may be used to determine social and political policies that can reduce risk for all forms of psychopathology at the population level.

The existence of a $\mathrm{P}$ factor has additional theoretical and clinical implications. First, identification of an individual's score on the P dimension can determine risk for all forms of psychopathology, while identification of one's Internalizing, Externalizing, and Thought Disorder scores can identify one's risk of specific forms of psychopathology, above and beyond that which is accounted for by P. It is possible therefore to develop a person-centred psychopathology profile that can be used to guide clinical decisions regarding monitoring, intervention, and treatment. Measures to assess the dimensions of psychopathology within the HiTOP model are currently under development however Kotov et al. (2017) provide useful suggestions for how clinicians can assess each dimension of psychopathology using existing measures. Second, as noted by Carragher et al. (Carragher et al., 2016), since the $\mathrm{P}$ factor is uncorrelated with the other dimensions of psychopathology, it is possible that an individual could display high levels of $\mathrm{P}$ but not be diagnosed with a specific psychiatric disorder should that person have correspondingly low levels on each specific dimension of psychopathology. Such an individual would however be at high-risk for the development of psychological distress and/or a psychiatric diagnosis. Construction of such person-centred psychopathology profiles offers a useful method to identify individuals who are asymptomatic but nonetheless at substantial risk for the development of psychopathology. Third, if $\mathrm{P}$ does indeed exist, comorbidity between psychiatric disorders - within and across dimensions of psychopathology - is inevitable. The WHO is currently preparing their 11th version of the International Classification of Diseases (ICD-11), and a stated objective of these revisions is to reduce diagnostic comorbidity by revising the symptom profiles of given diagnoses to ensure less symptomatic overlap between diagnoses (First et al., 2015). From a dimensional perspective of psychopathology this effort is in vain as the symptoms that comprise diagnoses, no matter how precisely defined and delineated, will all share at least one common underlying latent construct $(\mathrm{P})$ and possibly two (if two diagnoses are contained within the same specific dimension of psychopathology). This shared underlying latent construct(s) guarantees that comorbidity will occur. Fourth, effective treatments for a psychiatric disorder within a specific dimension of psychopathology (e.g., Cognitive Behaviour Therapy or selective serotonin reuptake inhibitors for major depressive disorder) should be similarly effective for other disorders within the same dimension of psychopathology (both interventions mentioned previously should be effective for PTSD, for example). Moreover, such treatments could also be expected to demonstrate efficacy for psychiatric disorders within other dimensions of psychopathology (e.g., schizophrenia) given that correlations can exist between dimensions. Indeed, this pattern of treatment efficacy is observed in the clinical literature (David et al., 2017). Fifth, treatment interventions that favour a transdiagnostic approach may be preferable to more diagnostic-specific interventions in order to reduce overall psychopathology.

Present findings should be considered in relation to several important limitations. First, as the current sample were predominately female CSA survivors from Denmark, the generalisability of these results to other clinical populations is limited. Second, the composition of the Externalizing dimension was limited, and the prevalence of substance dependence among this sample was low. Variation in the availability, selection, and/or inclusion of specific disorders across dimensional studies has been a recognised limitation of factor analytic research regarding psychopathology for some time (Uher and Rutter, 2012). Third, the use of the MCMI-III is a less than optimal method of assessing psychiatric diagnoses. Clinician-administered diagnostic interviews would have provided a methodologically superior means of diagnostic assessment, however, it is important that the structure of psychopathology be assessed using self-report assessments with clinical patients.

In conclusion, the findings of the current study provide initial evidence of the presence of a general factor of psychopathology, and its correlates, within a clinical sample. With a growing evidence base supporting the construct validity of $\mathrm{P}$, theoretical and clinical work may now benefit from identification of unique and shared risk-factors of each dimension of psychopathology. This offers the potential to develop person-centred risk-profiles for different patterns of psychopathology than can be used to guide clinical interventions. It is important to note however that while these dimensional models of psychopathology offer relatively parsimonious descriptions of disorder/symptom occurrence and co-occurrence from a research perspective, they may be more challenging to integrate within a clinical context. Many decisions in 
clinical practice are categorical. Clinicians must make judgements about whether to admit, treat, prescribe, and refer. The introduction of a dimensional score to inform such decisions is possible but far from straightforward (Allardyce et al., 2007). Dimensional measures with empirically derived cut-off points may in time outperform established diagnostic categories and improve clinical decision-making however this has yet to be demonstrated. Dimensional models have advanced representations of psychopathology from discrete yet substantially overlapping disorders to more general dimensions of psychological distress. In doing so, these dimensional models offer parsimonious accounts of distressing psychological experiences but have in turn also created an equally perplexing general factor of psychopathology to contend with.

\section{References}

Allardyce, J., Suppes, T., Van Os, J., 2007. Dimensions and the psychosis phenotype. Int. J. Methods Psychiatr. Res. 16 (Suppl 1), S34-S40.

American Psychological Association, 1994. Diagnostic and Statistical Manual of Mental Disorders, 4th ed. Author, Washington DC.

American Psychological Association, 2013. Diagnostic and Statistical Manual of Mental Disorders, 5th ed. Author, Washington DC.

Bebbington, P., 2009. Childhood sexual abuse and psychosis: aetiology and mechanism. Epidemiol. e Psichiatr. Soc. 18, 284-293.

Bendall, S., Jackson, H.J., Hulbert, C.A., McGorry, P.D., 2008. Childhood trauma and psychotic disorders: a systematic, critical review of the evidence. Schizophr. Bull. 34, 568-579.

Carragher, N., Teesson, M., Sunderland, M., Newton, N.C., Krueger, R.F., Conrod, P.J., Barrett, E.L., Champion, K.E., Nair, N.K., Slade, T., 2016. The structure of adolescent psychopathology: a symptom-level analysis. Psychol. Med. 46, 981-994.

Caspi, A., Houts, R.M., Belsky, D.W., Goldman-Mellor, S.J., Harrington, H., Israel, S., Meier, M.H., Ramrakha, S., Shalev, I., Poulton, R., Moffitt, T.E., 2014. The p factor: one general psychopathology factor in the structure of psychiatric disorders? Clin. Psychol. Sci.: J. Assoc. Psychol. Sci. 2, 119-137.

Christiansen, D.M., Hansen, M., Elklit, A., 2014. Correlates of coping styles in an adolescent trauma sample. J. Child Adolesc. Trauma 7, 75-85.

Collins, N.L., 1996. Working models of attachment: implications for explanation, emotion and behavior. J. Pers. Soc. Psychol. 71, 810-832.

David, D., Cotet, C., Matu, S., Mogoase, C., Stefan, S., 2017. 50 years of rational-emotive and cognitive-behavioral therapy: a systematic review and meta-analysis. J. Clin. Psychol.

Elklit, A., 1996. Coping styles questionnaire: a contribution to the validation of a scale for measuring coping strategies. Personal. Individ. Differ. 21, 809-812.

Elklit, A., 2004. En afprøvning af MCMI-III på et dansk patientmateriale. In: Elklit, A., Simonsen, D. (Eds.), En Introduktion til Millon Clinical Multiaxial Inventory, 2nd ed. Danish Psychology Press, Copenhagen, pp. 73-110.

Elklit, A., Shevlin, M., Solomon, Z., Dekel, R., 2007. Factor structure and concurrent validity of the world assumptions scale. J. Trauma. Stress 20, 291-301.

Enders, C.K., Bandalos, D.L., 2001. The relative performance of full information maximum likelihood estimation for missing data in structural equation models. Struct. Equ. Model.: Multidiscip. J. 8, 430-457.

First, M.B., Reed, G.M., Hyman, S.E., Saxena, S., 2015. The development of the ICD-11 clinical descriptions and diagnostic guidelines for mental and behavioural disorders. World Psychiatry.: Off. J. World Psychiatr. Assoc. (WPA) 14, 82-90.

Fleming, S., Shevlin, M., Murphy, J., Joseph, S., 2014. Psychosis within dimensional and categorical models of mental illness. Psychosis 6, 4-15.

Forbush, K.T., Watson, D., 2013. The structure of common and uncommon mental disorders. Psychol. Med. 43, 97-108.

Ireland, J.L., Boustead, R., Ireland, C.A., 2005. Coping style and psychological health among adolescent prisoners: a study of young and juvenile offenders. J. Adolesc. 28, 411-423.

Janoff-Bulman, R., 1989. Assumptive worlds and the stress of traumatic events: applications of the schema construct. Social. Cogn. 7, 113-136.

Jonas, S., Bebbington, P., McManus, S., Meltzer, H., Jenkins, R., Kuipers, E., Cooper, C., King, M., Brugha, T., 2011. Sexual abuse and psychiatric disorder in England: results from the 2007 Adult Psychiatric Morbidity Survey. Psychol. Med. 41, 709-719.

Kotov, R., Chang, S.W., Fochtmann, L.J., Mojtabai, R., Carlson, G.A., Sedler, M.J., Bromet, E.J., 2011a. Schizophrenia in the internalizing-externalizing framework: a third dimension? Schizophr. Bull. 37, 1168-1178.

Kotov, R., Krueger, R.F., Watson, D., Achenbach, T.M., Althoff, R.R., Bagby, R.M., Brown, T.A., Carpenter, W.T., Caspi, A., Clark, L.A., Eaton, N.R., Forbes, M.K., Forbush, K.T.,
Goldberg, D., Hasin, D., Hyman, S.E., Ivanova, M.Y., Lynam, D.R., Markon, K., Miller, J.D., Moffitt, T.E., Morey, L.C., Mullins-Sweatt, S.N., Ormel, J., Patrick, C.J., Regier, D.A., Rescorla, L., Ruggero, C.J., Samuel, D.B., Sellbom, M., Simms, L.J., Skodol, A.E., Slade, T., South, S.C., Tackett, J.L., Waldman, I.D., Waszczuk, M.A., Widiger, T.A., Wright, A.G.C., Zimmerman, M., 2017. The Hierarchical Taxonomy of

Psychopathology (HiTOP): a dimensional alternative to traditional nosologies. J. Abnorm. Psychol. 126, 454-477.

Kotov, R., Ruggero, C.J., Krueger, R.F., Watson, D., Yuan, Q., Zimmerman, M., 2011b. New dimensions in the quantitative classification of mental illness. Arch. General. Psychiatry 68, 1003-1011.

Krueger, R.F., Markon, K.E., 2011. A dimensional-spectrum model of psychopathology: progress and opportunities. Arch. General. Psychiatry 68, 10-11.

Laceulle, O.M., Vollebergh, W.A.M., Ormel, J., 2015. The structure of psychopathology in adolescence. Clin. Psychol. Sci. 3, 850-860.

Lahey, B.B., Applegate, B., Hakes, J.K., Zald, D.H., Hariri, A.R., Rathouz, P.J., 2012. Is there a general factor of prevalent psychopathology during adulthood? J. Abnorm. Psychol. 121, 971-977.

Lahey, B.B., Krueger, R.F., Rathouz, P.J., Waldman, I.D., Zald, D.H., 2017. A hierarchical causal taxonomy of psychopathology across the life span. Psychol. Bull. 143, $142-186$.

Lahey, B.B., Rathouz, P.J., Keenan, K., Stepp, S.D., Loeber, R., Hipwell, A.E., 2015. Criterion validity of the general factor of psychopathology in a prospective study of girls. J. Child Psychol. Psychiatry, Allied Discip. 56, 415-422.

Martel, M.M., Pan, P.M., Hoffmann, M.S., Gadelha, A., do Rosario, M.C., Mari, J.J., Manfro, G.G., Miguel, E.C., Paus, T., Bressan, R.A., Rohde, L.A., Salum, G.A., 2017. A general psychopathology factor (P factor) in children: structural model analysis and external validation through familial risk and child global executive function. J. Abnorm. Psychol. 126, 137-148.

McNulty, J.L., Overstreet, S.R., 2014. Viewing the MMPI-2-RF structure through the Personality Psychopathology Five (PSY-5) lens. J. Pers. Assess. 96, 151-157.

Millon, T., Millon, C., Davis, R., Grossman, S., 2009. MCMI-III Manual Pearson Education, Minneapolis, MN.

Molnar, B.E., Buka, S.L., Kessler, R.C., 2001. Child sexual abuse and subsequent psychopathology: results from the National Comorbidity Survey. Am. J. Public Health 91, 753-760.

Murphy, J., Shevlin, M., Houston, J.E., Adamson, G., 2014. Modelling the co-occurrence of psychosis-like experiences and childhood sexual abuse. Social. Psychiatry Psychiatr. Epidemiol. 49, 1037-1044.

Muthén, B.O., 2007. Factor Scores in SPSS vs. MPlus.

Muthen, L.K., Muthen, B.O., 2013. MPlus Statistical Analysis with Latent Variables. Muthen and Muthen, Los Angeles.

O'Connor, M., Elklit, A., 2008. Attachment styles, traumatic events, and PTSD: a crosssectional investigation of adult attachment and trauma. Attach. Human. Dev. 10, 59-71.

Owens, G.P., Chard, K.M., 2003. Comorbidity and psychiatric diagnoses among women reporting child sexual abuse. Child Abuse Negl. 27, 1075-1082.

Patalay, P., Fonagy, P., Deighton, J., Belsky, J., Vostanis, P., Wolpert, M., 2015. A general psychopathology factor in early adolescence. Br. J. Psychiatry.: J. Ment. Sci. 207, $15-22$.

Raftery, A.E., 1995. Bayesian model selection in social research. Sociol. Methodol. 25, $111-163$.

Roger, D., Jarvis, G., Najarian, B., 1993. Detachment and coping: the construction and validation of a new scale for measuring coping strategies. Personal. Individ. Differ. 15, 619-626.

Schafer, J.L., Graham, J.W., 2002. Missing data: our view of the state of the art. Psychol. Methods 7, 147-177.

Sellbom, M., 2017. Mapping the MMPI-2-RF specific problems scales onto extant psychopathology structures. J. Pers. Assess. 99, 341-350.

Tackett, J.L., Lahey, B.B., van Hulle, C., Waldman, I., Krueger, R.F., Rathouz, P.J., 2013. Common genetic influences on negative emotionality and a general psychopathology factor in childhood and adolescence. J. Abnorm. Psychol. 122, 1142-1153.

Uher, R., Rutter, M., 2012. Basing psychiatric classification on scientific foundation: problems and prospects. Int. Rev. Psychiatry (Abingdon, Engl.) 24, 591-605.

Waldman, I.D., Poore, H.E., van Hulle, C., Rathouz, P.J., Lahey, B.B., 2016. External validity of a hierarchical dimensional model of child and adolescent psychopathology: tests using confirmatory factor analyses and multivariate behavior genetic analyses. J. Abnorm. Psychol. 125, 1053-1066.

World Health Organization, 1992. The ICD-10 Classification of Mental and Behavioural Disorders: Clinical Descriptions and Diagnostic Guidelines. World Health Organization, Geneva.

Wright, A.G., Krueger, R.F., Hobbs, M.J., Markon, K.E., Eaton, N.R., Slade, T., 2013. The structure of psychopathology: toward an expanded quantitative empirical model. J. Abnorm. Psychol. 122, 281-294.

Yuan, K.H., Bentler, P.M., 2000. Three likelihood-based methods for mean and covariance structure analysis with nonnormal missing data. Sociol. Methodol. 30, 165-200. 